Document downloaded from:

http://hdl.handle.net/10251/102203

This paper must be cited as:

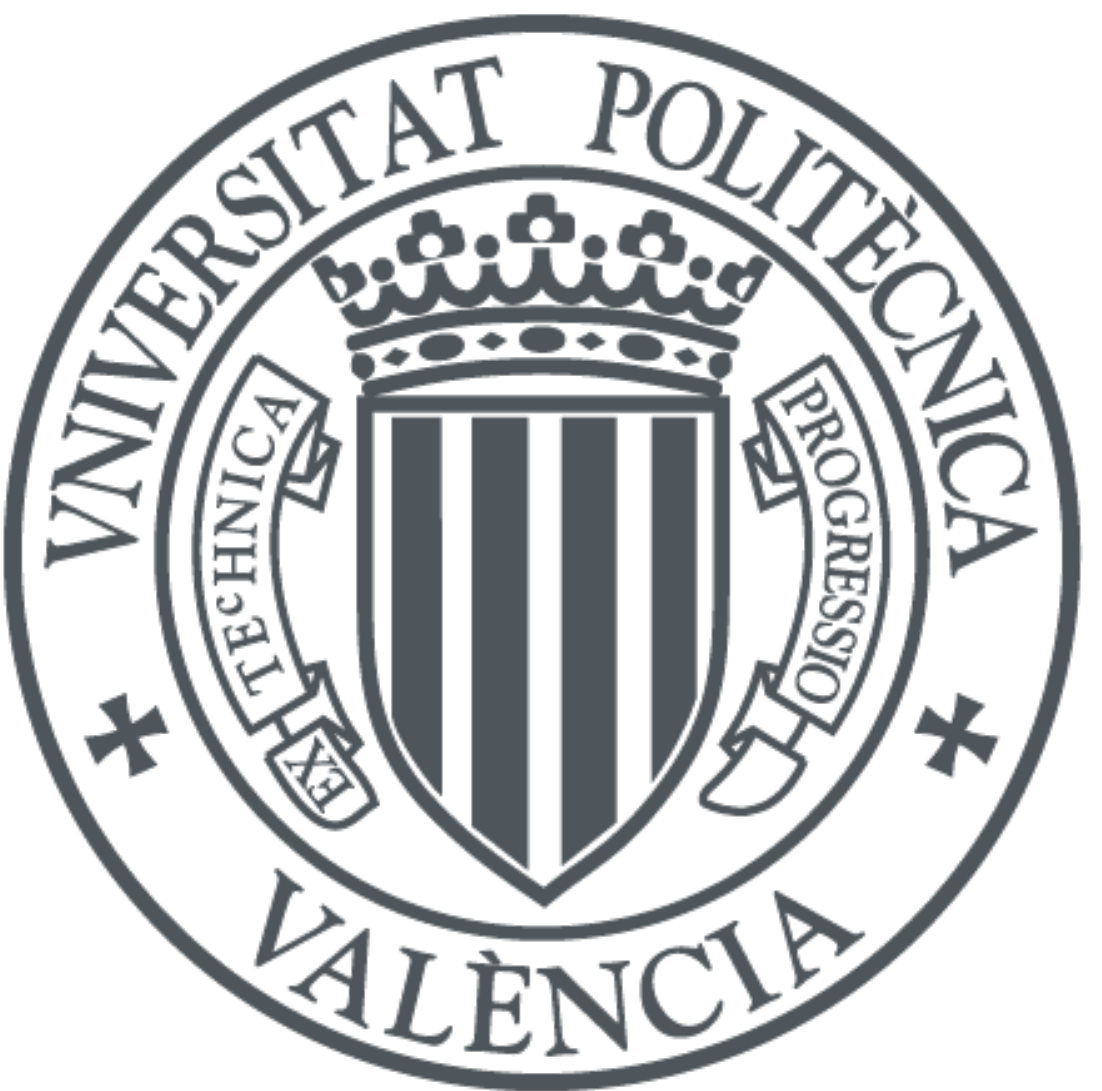

The final publication is available at

https://doi.org/10.1016/j.expthermflusci.2017.07.007

Copyright Elsevier

Additional Information 


\title{
Local flow measurements in a turbocharger compressor inlet
}

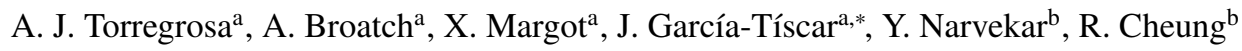 \\ ${ }^{a}$ CMT - Motores Térmicos, Universitat Politècnica de València, Camino de Vera, 46022 Valencia, Spain \\ ${ }^{b}$ Jaguar Land Rover Limited, Abbey Road, Whitley, Coventry CV3 4LF, UK
}

\begin{abstract}
This paper describes an experimental study carried out with the objective of characterizing flow instabilities in turbocharger compressors, specially the distribution of the high-temperature compressed backflow that appears upstream of the impeller at marginal surge conditions. The inlet of a test compressor was fitted with linear and circumferential thermocouple arrays in order to measure the temperature distribution caused by this backflow, whose independence of duct wall temperature was validated through thermographic imaging. Miniaturized pressure probes at the inducer and diffuser showed how pressure spectra varied during the different operating conditions. In-duct acoustic intensity was measured in both the inlet and the outlet to investigate the correlation between a known super-synchronous broadband issue known as whoosh noise and the backflow behaviour as characterized by local pressure and temperature. Analysis of the results points to inlet whoosh noise being boosted by this reversed flow but not caused by it, the source probably being located at or downstream of the compressor impeller.
\end{abstract}

Keywords: Aeroacoustics, Noise, NVH, Automotive, Whoosh, Surge

\section{Introduction}

As pressure grows on automotive internal combustion engines to meet ever increasing efficiency and sustainability requirements, engine manufacturers are resorting to further downsizing, with three-cylinder engines quickly replacing four-cylinder engines in many market segments.

Such a high reduction in the displacement due to environmental and efficiency concerns, implies the necessity of more aggressive turbocharging strategies in order to keep the engine power output at the value demanded by the vehicle [1]. These increased turbocharging requirements, together with strict packaging restrictions, have led to small turbocharger compressors operating at more demanding conditions, away from their stable operating points and closer to their surge limit [2].

Thoroughly studied for almost a century [3], deep surge is a destructive condition caused by airflow completely detaching from the compressor impeller blades, so that the compressed air reverses its direction and flows back from the diffuser to the inlet duct of the compressor. There, the momentum associated with the incoming fresh air motion forces the high temperature, reversed flow back into the compressor, and the detachment and reversing cycle starts again. This self-sustained cyclic phenomenon creates extreme pressure gradients that can compromise the structural integrity of the compressor. Hence, it is very important to avoid reaching such extreme operating conditions.
Different deep surge mitigation strategies have been proposed, either based on the modification of the inlet line and the compressor inducer [4, 5], or by introducing swirling [6] or pulsations [7] into the flow. These have been successful in delaying the onset of deep surge, thus expanding the useful air flow range of the compressor.

However, deep surge does not usually happen all of a sudden, especially in centrifugal compressors [3]. Before conditions become so critical that flow completely separates from the blades, partial detachments along the external edges of the blades begin to occur, leading to high temperature flow reversing along the periphery of the inducer and into the first portion of the inlet line.

Since turbocharger compressors keep decreasing in size to fit to smaller engines, their rotating speed further increases, while the minimum mass flow demanded by the engines keeps decreasing. As a result, operation in these partially stalled conditions is becoming increasingly common.

While the effect of partial blade stall on compressor performance and efficiency is well known (specially in axial flow compressors) since at least the 1950s [8,9], pressure disturbances caused by these "marginal surge" conditions have also been linked to increased noise generation in the turbocharging system. These Noise, Vibration and Harshness (NVH) issues are specially challenging in the development of modern downsized engines [10].

Regarding noise issues, not only an increase in the overall acoustic level is adversely perceived by the end-user, but also the subjective perception of noise quality is influenced by the frequency content of the acoustical emission. High speed tur- 


\section{Nomenclature}

+ Forward-travelling variable

- Backward-travelling variable

$\alpha$ Significance level (-)

$\bar{P}$ RMS averaged pressure $(\mathrm{Pa})$

X Pressure wave spectrum $(\mathrm{Pa})$

$\rho$ Ambient density $\left(\mathrm{kg} \mathrm{m}^{-3}\right)$

$a$ speed of sound $\left(\mathrm{m} \mathrm{s}^{-1}\right)$

$B$ Greitzer-Moore $B$ parameter (-)

$D$ compressor inlet diameter $(\mathrm{m})$

$f_{1} 1^{\text {st }}$ symmetric acoustic mode onset frequency $(\mathrm{Hz})$

$f_{2} 2^{\text {nd }}$ symmetric acoustic mode onset frequency $(\mathrm{Hz})$

$I$ Sound intensity $\left(\mathrm{W} \mathrm{m}^{-2}\right)$

$I_{0}$ Reference sound intensity $\left(\mathrm{W} \mathrm{m}^{-2}\right)$
$M$ Mach number (-)

$p_{0}$ Reference pressure $(\mathrm{Pa})$

AMF Corrected Air Mass Flow $\left(\mathrm{kg} \mathrm{s}^{-1}\right)$

BPF Blade Passing Frequency $(\mathrm{Hz})$

CAD Computer Aided Design

CFD Computer Fluid Dynamics

LCMV Linearly Constrained Minimum Variance

NVH Noise, Vibration and Harshness

RMS Root Mean Square

SIL Sound Intensity Level (dB)

SPL Sound Pressure Level (dB)

TCN Tip Clearance Noise bomachinery can add high frequency components to the usual low frequency sounds of piston engines, and thus negatively impact the customers' perception of the vehicle noise quality and performance [11].

In this paper, an experimental campaign is carried out with the double objective of characterizing the aforementioned hot backflow, from its inception at nearly stable conditions to its evolution until just before deep surge is reached, and studying its influence on noise generation.

Compressor instrumentation is described, with indication of the different sensors used and their locations. Selected results from the experimental campaign are presented and discussed, focusing on how the different thermodynamic results can be useful to characterize the evolution of stall and its relation to acoustical phenomena.

\section{Experimental setup}

The experiments were carried out in a large anechoic chamber available at Instituto CMT - Motores Térmicos. More details about the characteristics of this facility and its use for turbocharger research can be found in [12] and [13], respectively.

\subsection{Turbocharger}

For this investigation, a production automotive turbocharger was chosen. However, prior to its installation in the gas stand, modifications were made to accommodate the required local temperature and pressure sensors.

The compressor housing was modified by removing the original inducer up to the plane of the blade leading edges and substituting it by a removable adapter piece which incorporates guides for a circumferential array of thermocouples and a miniaturized pressure probe. This adapter is depicted in red in Figs. 1 and 2 .

A detachable straight pipe section with a linear thermocouple guide was affixed to this adapter. This configuration offers the possibility of easily substituting the straight section for other ones with different geometries such as elbows or tapered ducts.

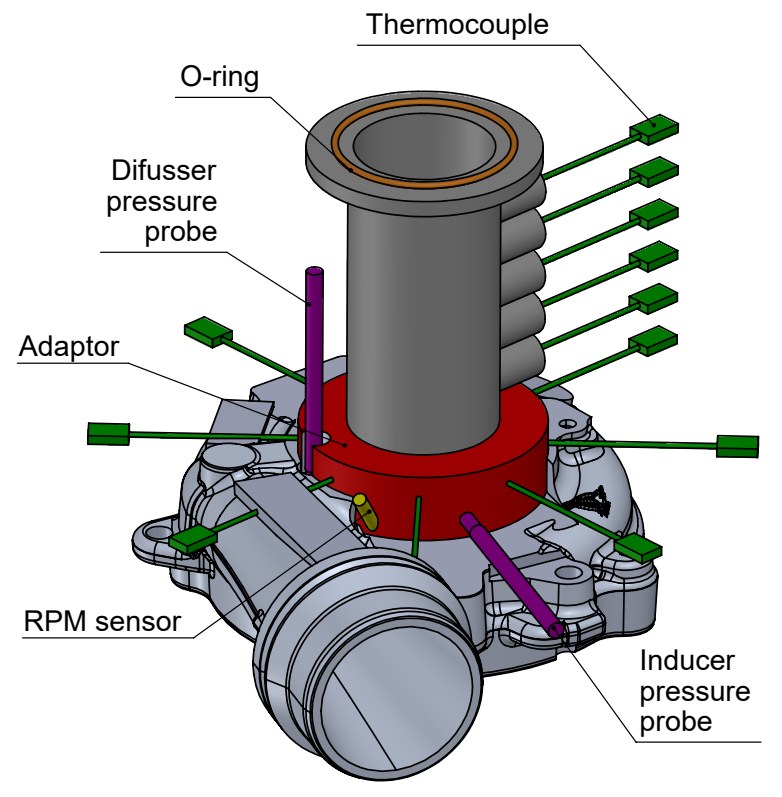

Figure 1: CAD view of the proposed local temperature and pressure measurement instrumentation, showing the distribution of thermocouple arrays and pressure probes.

\subsection{Temperature}

It is well established in the literature $[14,15]$ that flow reversing from downstream of the impeller is one of the most significant phenomena indicating compressor instability and stall inception. Since these backflows coming from downstream of the wheel have undergone the non-isentropic compression process, they are at high temperature. Consequently, not only pressure but also temperature measurements should be considered for characterizing both the inception of these backflows and their upstream extension. Indeed, Liu et al. [16] for instance showed how the standard deviations of temperature and pressure measurements are good indicators of deep surge. 
Two thermocouple arrays were thus installed at the compressor inlet in order to characterize the temperature distribution associated with this backflow. A circumferential array covers a cross section of the pipe just at $1 / 3$ inlet diameters, or $14 \mathrm{~mm}$ from the compressor wheel plane, providing temperature measurements every $45^{\circ}$. The second array is linear, extending in the longitudinal direction of the pipe up to approximately 2 inlet diameters. Both arrays can be seen in Fig. 1 and especially in Fig. 2.

The first array was devised to determine if there existed angular variations of temperature distribution indicating thus the presence of some backflow, and the second one was used to characterize the length of the recirculating backflows for each compressor operating condition.
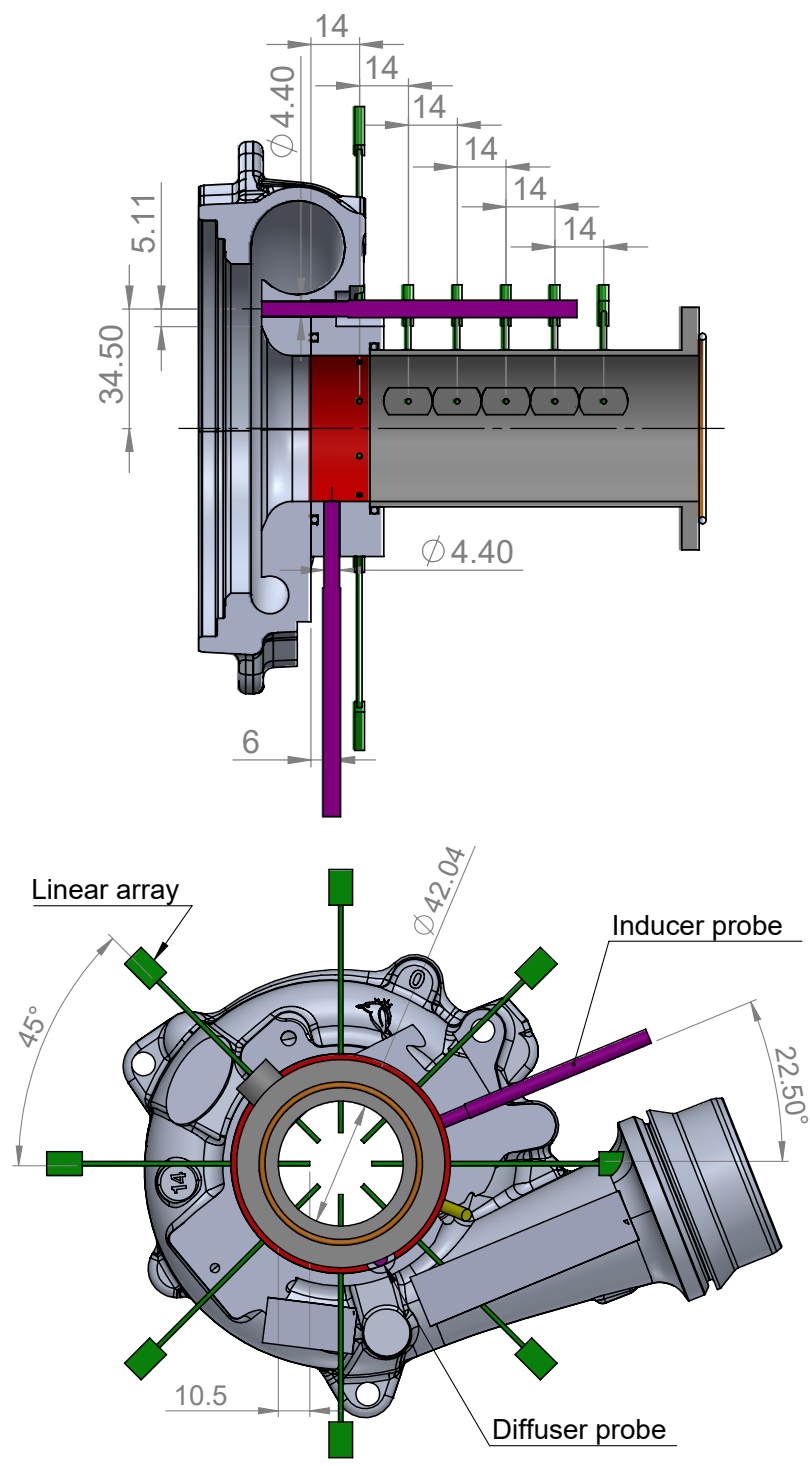

Figure 2: Annotated views of the pressure probes (purple) and thermocouples (green) locations, forming the circumferential and linear arrays.
Numerical simulations performed by Lang [17] suggest that backflows extend up to $2-3$ diameters in length, and may occupy over half of the duct radius area. In order to characterize the backflow, Andersen et al. [18] selected a distance of half the duct radius for placing several thermocouples in the inlet pipe of the compressor, whereas Figurella et al [19] used thermocouples located in the centre of the pipes and an additional one $14 \mathrm{~mm}$ away from the blade tips and protruding $4 \mathrm{~mm}$ into the duct.

Further temperature measurements were taken in order to provide reference points for the two arrays. The ambient temperature of the test chamber was recorded, as this is the temperature of the ingested flow. A surface thermocouple was also affixed to the external wall of the aforementioned circular array adapter. All the thermocouple measurements were acquired with a Yokogawa oscilloscope, and were averaged over one second.

Additionally, a thermographic camera was installed onto the turbocharger assembly, so that its view frame covered both the compressor volute and the inlet duct. This provided a measurement of the external distribution of temperature, that proved useful to confirm that the temperature profiles measured by the linear thermocouple array were not affected by heat transfer from the inlet pipe walls.

\subsection{Pressure}

Pressure measurements are usually taken in both the inlet and the outlet ducts of the compressor. Within this work, an effort was made to characterize the spectral signature of the pressure at two additional critical locations: in the inducer, upstream of the compressor wheel, and in the diffuser, downstream of the wheel. Miniaturized piezoelectric pressure probes from Kistler were used in both locations.

The inducer probe was mounted flush to the wall, at a distance of $6 \mathrm{~mm}$ from the wheel leading edge to the centreline of the sensor. The diffuser probe was mounted at $5 \mathrm{~mm}$ from its inlet section and $34.5 \mathrm{~mm}$ away from the turbocharger axis line. Both probes can be seen in Fig. 1 and Fig. 2.

\subsection{Noise}

In addition to the local measurements near the compressor inlet, sound intensity was measured in both the inlet and the outlet ducts, in order to relate the evolution of the local inlet flow field to the sound emission. Specifically, a broadband noise known in the literature [20, 21, 22] as "whoosh noise" was targeted, since it has been associated with local instabilities near the impeller.

The methodology chosen for measuring sound intensity is based on pressure wave decomposition, so that the effect of mean flow velocity on sound propagation is accounted for. A detailed step-by-step explanation can be found in [23].

Decomposition of the pressure into forward- and backwardpropagating waves was performed using a Linearly Constrained Minimum Variance (LCMV) beamforming algorithm on the data recorded by two linear arrays of three flush-mounted piezoelectric pressure transducers installed in both the inlet and the outlet pipes. 


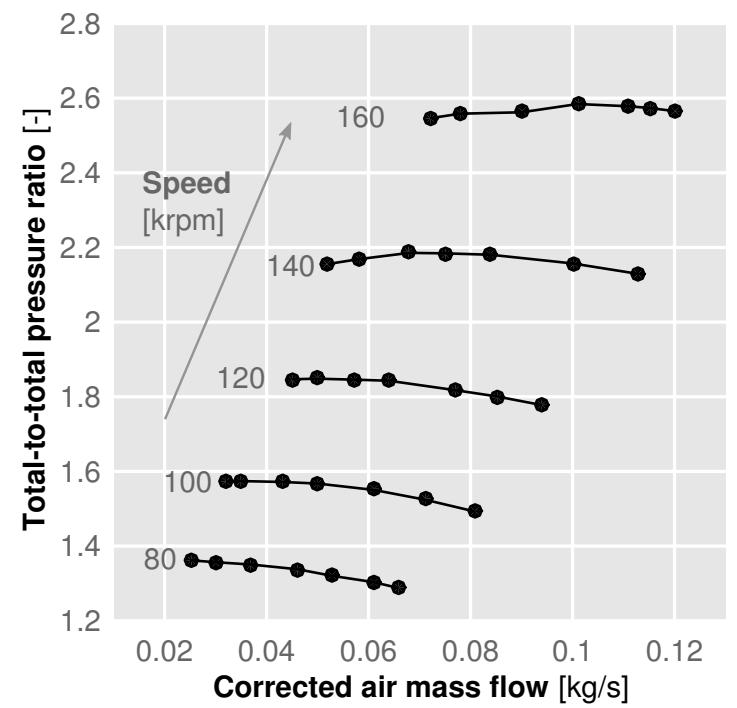

Figure 3: Compressor map showing the operating conditions covered during the measurement campaign and the slope of the characteristic speed curves that indicate the onset of stall where the slope changes its sign. From top to bottom, shaft speeds were 160,140,120, 100 and $80 \mathrm{krpm}$.

The three sensor arrays were installed at suitable distances from the compressor where the flow could be considered fully developed and where it should be possible to avoid $1 / 3$ and $1 / 5$ standing wave nodes as recommended by Piñero et al. [24]. The distance between sensors was chosen so as to provide a measurement range of up to $3 \mathrm{kHz}$, consistent with previous experience of whoosh noise results [25, 26, 27].

\section{Results and discussion}

Using this setup, data was captured at several turbocharger operating conditions. Different shaft speeds were kept constant while air mass flow was progressively reduced, from a stable operating point close to the compressor maximum efficiency region to an unstable point very close to the compressor deep surge limit. Figure 3 shows the recorded points.

In order to prevent the appearance of deep surge, with its inherent danger to the integrity of the compressor, pressure signals were closely monitored to detect the onset of very low frequency components ( 5 to $20 \mathrm{~Hz}$ ) which were shown by Galindo et al. [28] to be significant indicators of deep surge. It should be noted however that the precise frequencies vary with the compressor and inlet geometry and should be either measured or estimated through the Helmholtz frequency and the $B$ parameter as discussed by Fink et al. [29].

\subsection{Temperature}

\subsubsection{Radial profile}

Prior to the measurements of the whole compressor chart, a sweep of temperature measurements along the inlet radius was performed for both the thermocouple located at $1 / 3 \times D$ and the thermocouple located at $2 \times D$ from the leading edge. For these measurements, the turbocharger compressor was brought to conditions close to deep surge at $80 \mathrm{krpm}$.

The results can be seen in Fig. 4. As expected, the overall temperature profile is higher at $1 / 3 \times D$ from the leading edge plane than at $2 \times D$. The temperature appears to be approximately constant up to a distance of $1 / 8$ diameters $(5 \mathrm{~mm})$ from the internal wall, then decaying up to a distance of approximately $1 / 4$ diameters $(10.5 \mathrm{~mm})$ from the centreline, which is at $1 / 2$ diameters or $21 \mathrm{~mm}$ from the wall. From there onwards, temperature is again approximately constant.

Ambient temperature and surface temperature of the adapter (seen in Figs. 1 and 2 in red) can also be seen in Fig. 4. As expected, the centreline temperature at the most upstream point is almost coincident with that of the ambient, while the fluid temperatures near the wall at the most downstream point (at just $1 / 3 \times D$ from the wheel) are almost equal to those of the adapter external wall, save for a small initial difference due to heat losses.

From these results it is apparent that measuring the fluid temperature in the vicinity of the wall is not practical due to heat transfer from the wall to the thermocouple. Standardized procedures for the characterization of flow temperature call for measurements at $1 / 4$ and $1 / 6$ of the diameter. In this case, this would correspond to distances of $7 \mathrm{~mm}$ and $10.5 \mathrm{~mm}$ away from the wall. It is indeed clear on Fig. 4 that at these distances the temperature values differ significantly from wall-influenced temperature values. Ultimately, a distance of $1 / 4$ of the diameter $(10.5 \mathrm{~mm})$ was chosen for this study in accordance with the work of Andersen et al. [18].

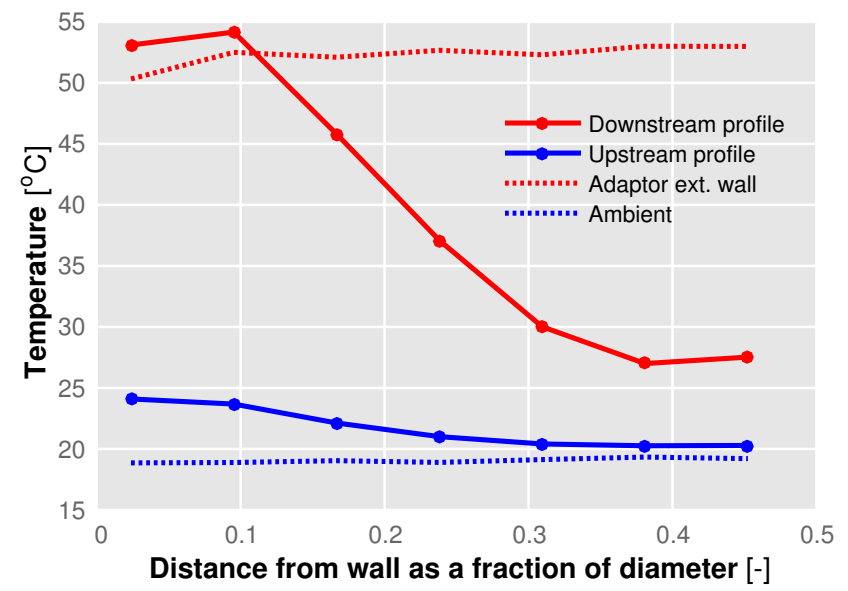

Figure 4: Evolution of temperature measured by the first and last thermocouples of the linear array for different distances from the wall, including measurements of ambient temperature of the chamber and surface temperature of the adapter. 
Figure 4 also shows that even at the core of the flow there is a rise in temperature of about 8 degrees between the upstream and the downstream profiles. Even though the hot backflow is expelled towards the walls, its high rotational speed favours mixing with the cold core flow and thus heat diffusion, which explains the core flow temperature increase observed along the whole profile.

\subsubsection{Backflow growth}

By analyzing the evolution of the temperature results in the inlet duct for different operating conditions (speed and air mass flow) it is possible to estimate the growth of the recirculating backflow.

Figure 5 shows different profiles of temperature difference with respect to the ambient, measured by the linear thermocouple array for the same rotating speed and different mass flows. They show that at higher mass flows the temperature along the inlet pipe is approximately equal to that of the ambient.

At low mass flow rates, the flow instabilities inside the wheel channels cause the reversal of the air heated by the compression process, so that a temperature increase coming from the compressor wheel starts to be noticeable. The backflow onset seems to occur at an operating condition corresponding approximately to that of maximum pressure ratio at each rotating speed, as seen in Fig. 3.

The temperature evolution along the inlet duct differs clearly depending on the rotating speed. At low rotating speeds, the backflow reaches farther upstream than 2 diameters from the wheel, especially at low air mass flow rates, but the maximum temperature difference is lower. Conversely, at high rotating speeds the maximum temperature difference is larger (due to the higher pressure ratio) but the extension of the backflow along the inlet pipe is more limited.

The shrinkage of the backflow length at high rotating speed can probably be attributed to higher inlet flow momentum at these operating conditions. Reduced inlet flow momentum at low shaft speeds would instead allow the compressed hot backflow to reach farther upstream into the inlet line.

In order to ensure that the temperature increase along the inlet pipe measured with the thermocouples is effectively caused by backflow-convected heat and not by thermal diffusion from the heated compressor housing to the metallic inlet pipe, an additional test was performed whereby thermographic images of the compressor were captured during the measurements, as shown in Fig. 6.

These snapshots were synchronized with the operating conditions at which flow temperatures were measured inside the duct. Inlet pipe radiated wall temperature profiles were extracted to compare the temperature rise to that of the internal flow. Figure 7 shows this comparison.

Considering first the highest mass flow operating condition (blue lines) where there is no backflow exiting from the compressor, it is apparent that while the wall temperature follows a typical heat diffusion process, i.e. the external wall temperature profile (dotted line) decays continuously, the flow temperature measured by the thermocouples (solid line) remains constant along the pipe.
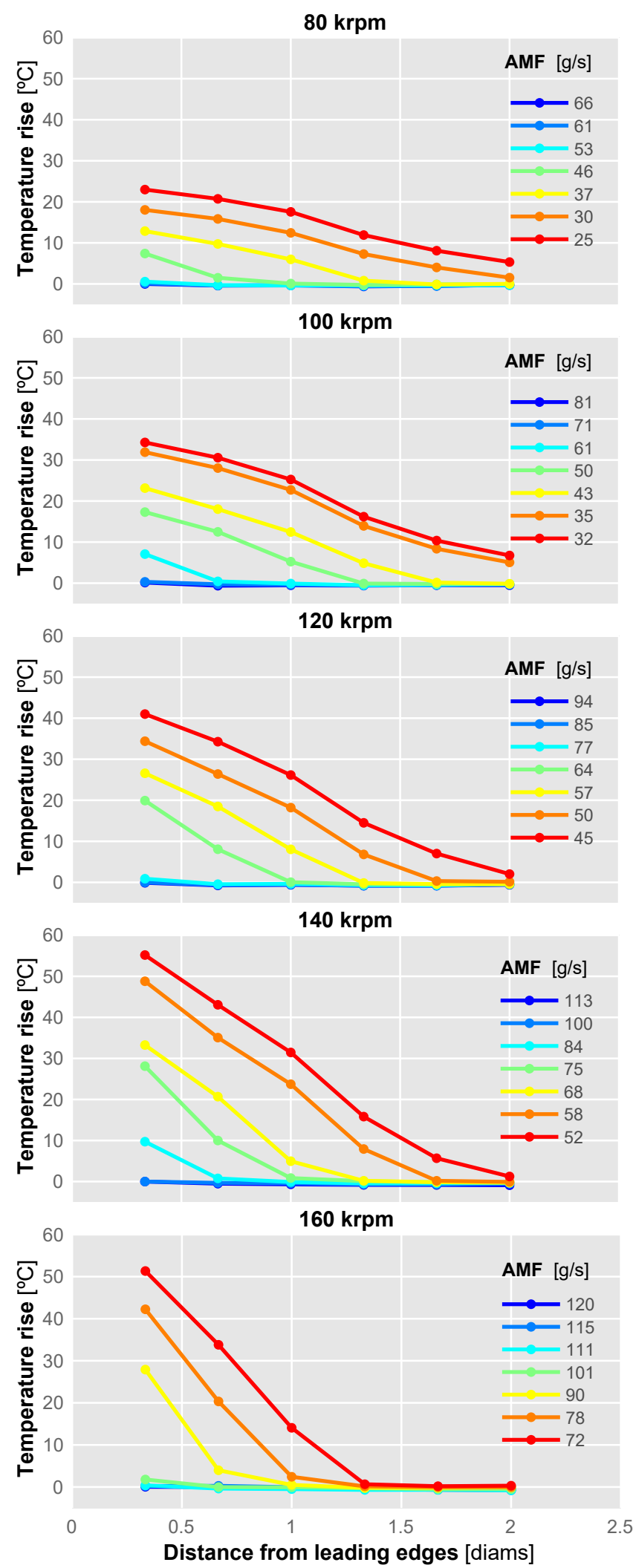

Figure 5: Evolution of temperature rise measured by the linear array thermocouples for different mass flow settings and different shaft speeds, taking the most upstream temperature as reference. 


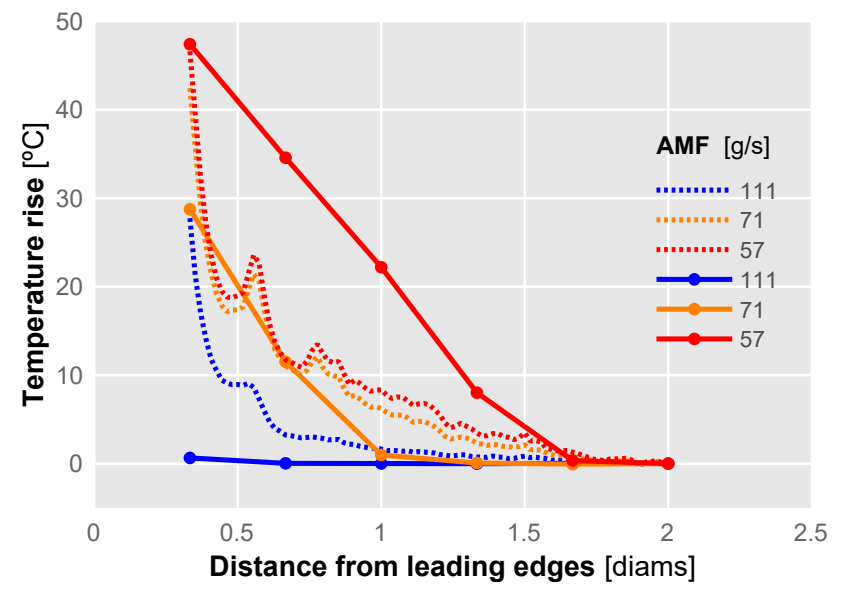

Figure 7: Comparison of the temperature rise measured by the thermographic camera (dotted line) and the internal thermocouples (solid line) for different mass flow settings, taking the most upstream temperature as reference.

At lower mass flow rate (orange lines) the effect of the backflow can be clearly observed, as the internal thermocouples register a temperature rise up to an upstream distance of 1 diameter. Further upstream of this point however, the flow temperature remains constant and equal to that of the most upstream point, whereas the external wall profile shows a rather continuous decay up to a distance of over 1.5 diameters.

As for the lowest mass flow condition (red lines) the temperature increase measured by the internal thermocouples is higher than the temperature registered by the thermographic camera, and so in this case heat would be transferred from the hot backflow to the walls and not the other way round.

\subsubsection{Circumferential profile}

As previously described, temperature measurements were also recorded using the circumferential thermocouple array situated $1 / 3 \times D$ away from the compressor leading edge. The objective in this case was to characterize the angular distribution of temperature near the impeller for different operating conditions.
The result of this study can be seen in Fig. 8, where the circumferential temperature profiles at each measured air mass flow value are plotted for the different rotating speeds considered. These air mass flow values were the same as those previously shown in Fig. 5 (where the linear array temperatures were plotted).

It can be observed that the skewness of the circumferential profile increases when the mass flow is reduced. At high flows, the skewness is practically zero, the profile being completely regular as the temperatures are similar for all angular positions. However, at the point of higher pressure ratio some skewness starts to appear along with an increase in temperature due to the hot backflow.

As the air mass flow is further decreased towards the compressor surge limit, an increase of both the overall temperature and the profile skewness may be observed. The pattern of the increased skewness appears to be similar for all the conditions considered: the temperature is higher at angles corresponding to the tongue and the end of the volute, where its cross-section is larger, and smaller at the angles where the volute cross-section is still small.

While minimum temperatures (corresponding to high air mass flows) remain similar for all rotating speeds and approximately equal to the ambient temperature, the maximum temperatures increase with rotating speed. This is consistent with the fact that the backflow is hotter due to the increased compression ratio.

However, the increased angular differences exhibited by these maximum temperatures are noticeable, with a difference between the $90^{\circ}$ and the $270^{\circ}$ angular positions of almost $20^{\circ} \mathrm{C}$ for the lowest air mass flow at $160 \mathrm{krpm}$ (red line in the rightmost plot of Fig. 8).

\subsubsection{Temperature compressor charts}

In order to visualize the variation of the previously presented temperature results with the compressor operating condition, selected results have been mapped onto the compressor chart presented in Fig. 3 by means of an interpolation procedure. The result of these mappings can be seen in Fig. 9.
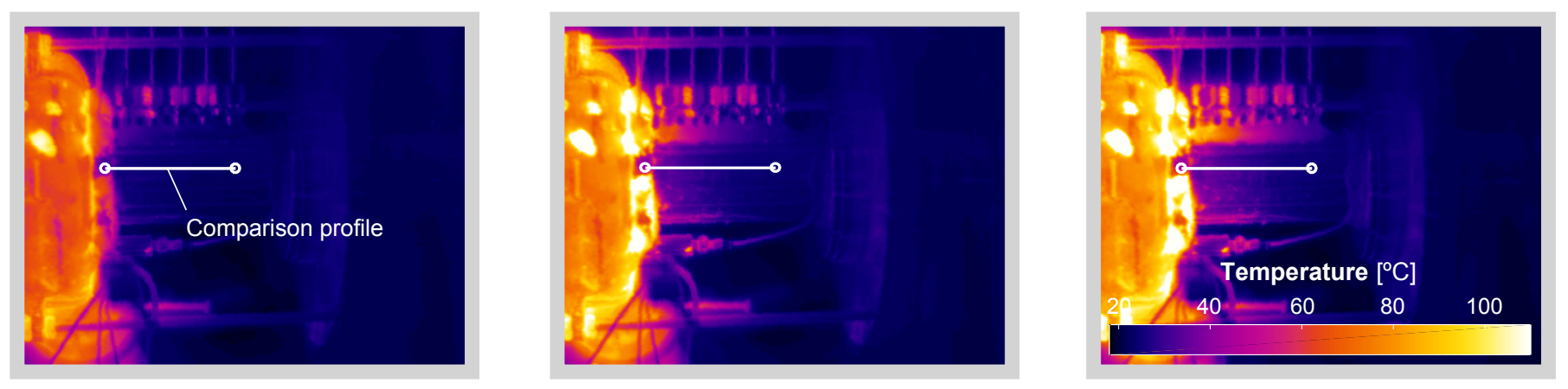

Figure 6: Thermographic (IR) measurement of the compressor at a rotating speed of $140 \mathrm{krpm}$ and different air mass flows, showing the progressive rise of wall temperature as air flow decreases. From left to right: 111,71 and $57 \mathrm{~g} / \mathrm{s}$. White line indicates the temperature profile used for comparison in Fig. 7. 


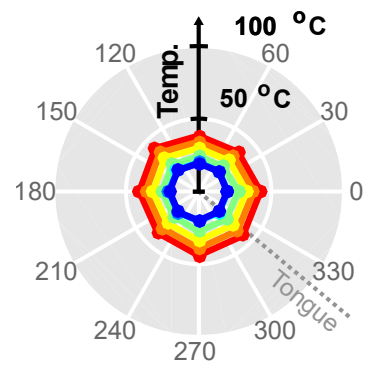

80 krpm

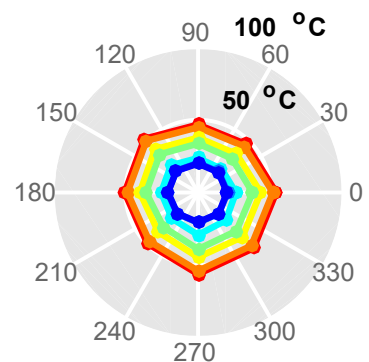

$100 \mathrm{krpm}$

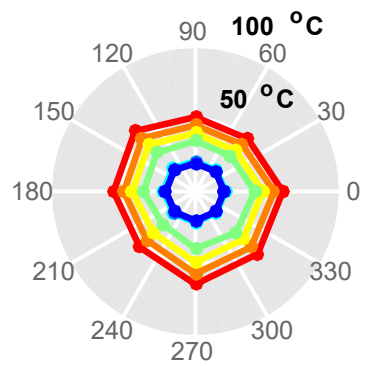

$120 \mathrm{krpm}$

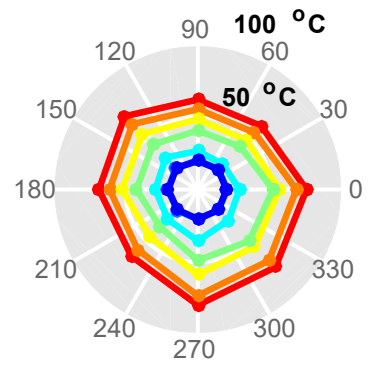

$140 \mathrm{krpm}$

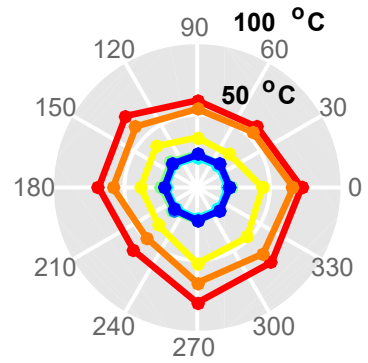

$160 \mathrm{krpm}$

Figure 8: Evolution of temperature measured by the circumferential array thermocouples as air mass flow was reduced keeping shaft speed constant. A polar temperature plot is shown for each speed, colors indicating mass flow from higher (blues) to lower (reds). Legends for colors at each speed are available in Fig. 5. See Fig. 2 for reference position of the compressor volute.
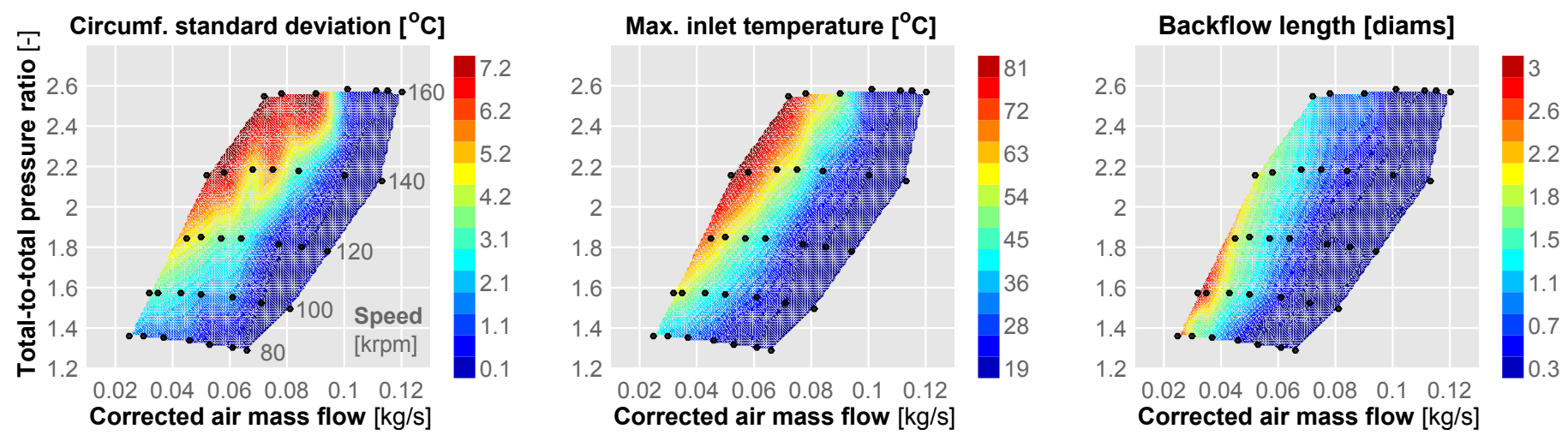

Figure 9: Maps summarizing the results obtained through temperature measurements across the different operating conditions. From left to right: circumferential temperature skewness, maximum temperature and estimated backflow length.

The leftmost chart shows that the skewness of the circumferential temperature distribution depends on the operating conditions. The standard deviation has been chosen as a metric to quantify the magnitude of the skewness. It can be seen that the skewness increases with rotating speed and the instability caused by low air mass flows.

In the centre chart of Fig. 9 the overall maximum temperature measured by the thermocouples is shown. The distribution along the chart is similar to that of the skewness albeit less pronounced at high speeds. At higher mass flows, temperature is very close to the ambient temperature $\left(\sim 20^{\circ} \mathrm{C}\right.$ or $\left.293 \mathrm{~K}\right)$ of the chamber. Maximum temperature in the array starts to increase once the slope of the iso-speed lines approaches zero. This temperature rise can reach up to $80^{\circ} \mathrm{C}$ at $160 \mathrm{krpm}$ and conditions close to surge.

Finally, an estimation of the backflow upstream extension is provided in the rightmost chart of Fig. 9. This was done by post-processing the raw data from the linear array displayed in Fig. 5. The temperature profile for each measurement point (rotating speed and air mas flow) was automatically processed with the procedure described below.
Starting from the thermocouple closest to the impeller, the reading of each thermocouple in the linear array is compared with the ambient temperature; the first that reads less than $3^{\circ} \mathrm{C}$ above the ambient is assumed to indicate the backflow spatial extension.

If this is not achieved, it could be due to the temperature profile being uniform. In order to check this condition, maximum temperature difference between all linear thermocouples is calculated. If this difference is smaller than $3^{\circ} \mathrm{C}$ a constant temperature profile has been detected. If the maximum temperature of the profile is less than $10^{\circ} \mathrm{C}$ above the ambient temperature, the backflow length is assumed to be minimum $(1 / 3 \times D)$, the deviation in temperature being attributed to prolonged previous operation at high temperature points. Else, the length is assumed to be maximum $(2 \times D)$, implying a very large backflow region. This situation only occurred when the compressor entered deep surge conditions. 

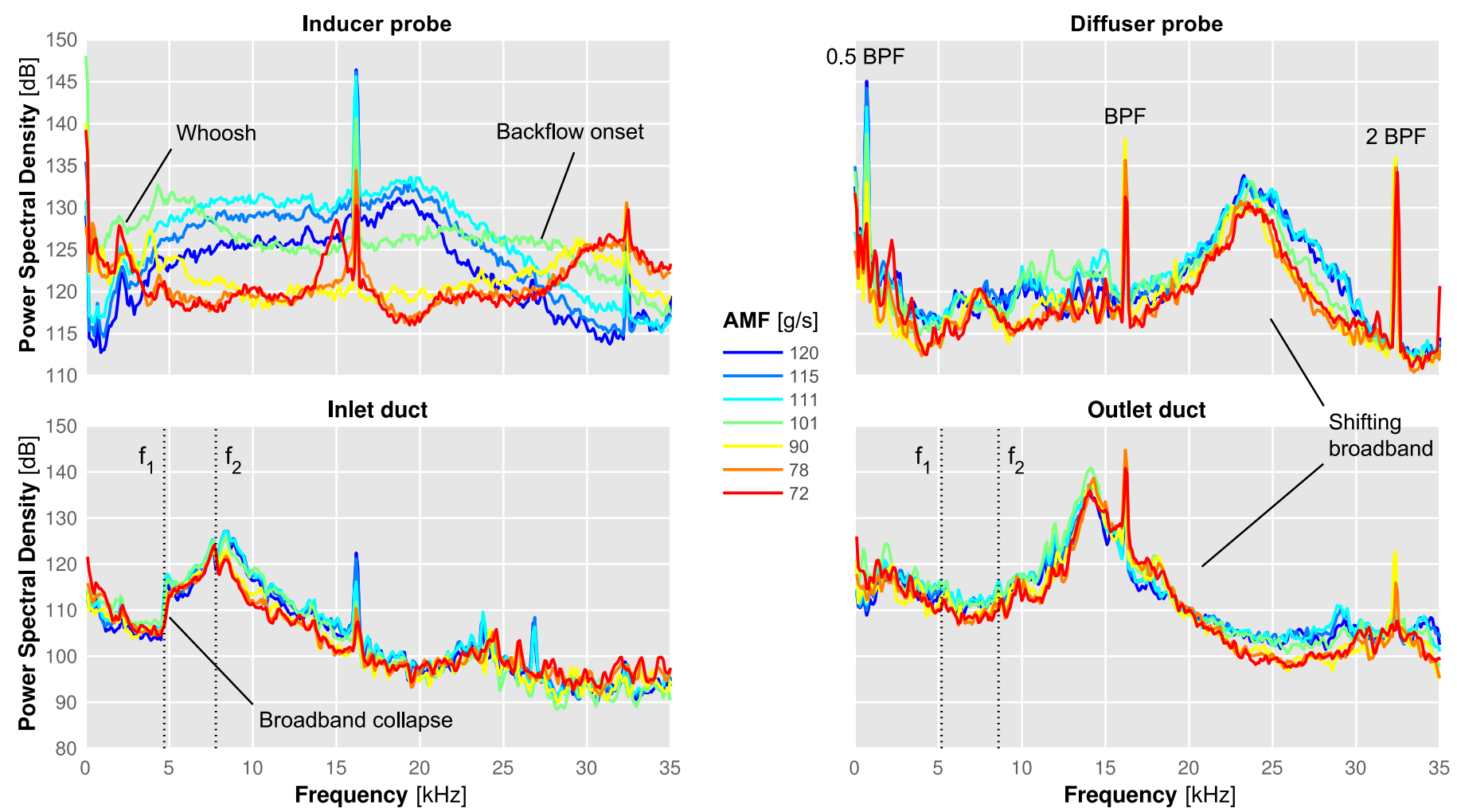

Figure 10: Top row: pressure spectra at the inducer (left) and diffuser (right) measured by the local pressure probes at different air mass flows while the shaft speed was kept constant. Bottom row: pressure spectra at the inlet (left) and outlet (right) ducts, including the onset of the first and second acoustic modes.

If the profile still does not satisfy the previous criteria, this means that the backflow is extending beyond the length of the linear thermocouple array, but still has a distinguishable slope. In this case a linear-log extrapolation of the measured profile was performed. The backflow maximum length is assumed to be located where the extrapolated profile reaches a value of $3^{\circ} \mathrm{C}$ above the ambient temperature.

The resulting chart plotted in Fig. 9 shows that no backflow is apparent at high mass flows, with its length only reaching the thermocouple array near the point of zero slope of the iso-speed line. However, contrary to the case of the previous two charts, the backflow length is higher at low rotating speeds, as lower mass flows provide less momentum to counteract the backflow.

\subsection{Pressure}

\subsubsection{Local pressure}

As shown in Figs. 1 and 2, miniature pressure probes were fitted in both the inducer and the diffuser of the compressor in order to characterize the behaviour of the pressure as the operating conditions change. In Fig. 10, the top row shows the sound pressure level spectra for both inducer and diffuser probes at a constant rotation speed of $160 \mathrm{krpm}$ and different air mass flows. The frequency range is from 0 to $35 \mathrm{kHz}$, an upper bound which is well beyond the human hearing range but nonetheless shows relevant information. As the behaviour for the rest of the tested rotational speeds was similar, the discussion will be presented only for this condition.
It can be seen in these two plots that the pressure spectra in the diffuser (right) are very similar, regardless of the different air mass flows considered, with a marked increase only for frequencies below $5 \mathrm{kHz}$ and above $15 \mathrm{~Hz}$, which will be explained below.

A broadband noise centered around $2 \mathrm{kHz}$ becomes apparent at low flow conditions, with two side lobes. A characteristic Blade Passing Frequency (BPF) can be clearly seen as a peak at $16 \mathrm{kHz}$, along with higher and lower frequency harmonics. This could be related to the generation of whoosh noise, which often identified in this frequency range.

Another very noticeable broadband noise is also present at higher frequencies, peaking at around $23 \mathrm{kHz}$, which is a higher frequency than BPF. This other broadband content could be related to the so-called Tip Clearance Noise (TCN) mentioned by Raitor and Neise [30] based on a model for axial turbomachinery presented by Kameier and Neise [31], and which was also reported by Tiikoja et at. [32]. Numerical studies by Galindo et al. [33] however do not appear to support the hypothesis of tip clearance being the leading mechanism.

Data from the inducer pressure probe on the left top plot shows very different spectra depending on the compressor operating condition. At high flow conditions, the frequency content below $5 \mathrm{kHz}$ is lower than the rest of the audible content between $5 \mathrm{kHz}$ and $22 \mathrm{kHz}$. A slight broadband can be seen peaking 
around $20 \mathrm{kHz}$. As the flow is reduced from 120 to 115 and 111 $\mathrm{g} / \mathrm{s}$ all the frequency content raises in level but the distribution is similar, with the broadband being more pronounced, but still peaking around the same $20 \mathrm{kHz}$ frequency.

As seen in Figs 9 and 5, the next measurement point at $101 \mathrm{~g} / \mathrm{s}$ is the first where the hot backflow reaches the first thermocouple at $1 / 3$ diameters from the impeller. Recall from Fig. 2 that the inducer pressure probe was located between this thermocouple and the impeller. Hence the fact that the backflow is reaching the thermocouple implies that the probe readings are starting to be affected by this backflow.

The backflow arrival causes a radical change in the pressure spectrum: in contrast with the previous operating point, at 101 $\mathrm{g} / \mathrm{s}$ (teal coloured line) the highest level is now located at and below $5 \mathrm{kHz}$ with a steady decrease up to $10 \mathrm{kHz}$ and a more or less constant content until the level starts to fall at $28 \mathrm{kHz}$ and above.

As the air mass flow was further decreased to $90 \mathrm{~g} / \mathrm{s}$, the backflow extended upstream to at least the second thermocouple at 1 diameter distance from the impeller leading edges.

Again, this changes the spectrum, with the higher levels now descending from 0 to $8 \mathrm{kHz}$, a constant level from 8 to approximately $25 \mathrm{kHz}$ and a broadband peaking at approximately 30 $\mathrm{kHz}$.

The following two operating points, where the air mass flow was still further reduced (to 78 and $72 \mathrm{~g} / \mathrm{s}$ ) and the backflow keeps extending upstream (as seen in the thermocouple readings presented in Fig. 5) exhibit spectra similar to those previously described, but with the high frequency broadband slightly shifting to reach approximately $32 \mathrm{kHz}$.

As in the case of the measurements from the diffuser pressure probe, the Blade Passing Frequency peak at $16 \mathrm{kHz}$ and its lower and higher frequency harmonics can be clearly distinguished at all operating points.

Lastly, the lower frequency broadband at around $1-3 \mathrm{kHz}$ that is usually associated with whoosh noise can be clearly distinguished at all mass flow conditions, specially in the inducer spectra, even at the highest mass flow rate for which the backflow has not yet reached the probe location.

This could be related to the phenomena previously observed by Liśkiewicz [34] where some frequencies characteristic of unstable fluid structures were shown to be present in damped form even at stable conditions, indicating in this case that whoosh is still being produced at more stable operating conditions.

\subsubsection{In-duct pressure}

In addition to the miniature pressure probes at the inducer and the diffuser of the compressor, pressure was also measured with piezoelectric transducer arrays in the straight inlet and outlet ducts. While these arrays were mainly used to compute plane wave sound intensity, in addition the spectrum of the first sensor of each array was plotted in the bottom row of Fig. 10 to compare the pressure signature in the ducts with those measured by the probes.
Comparing the pressure spectra measured in the outlet duct (bottom right plot) with the previously described spectra measured in the diffuser (top right plot) it can be seen that the results are similar except for the shifting of the high frequency broadband, from approximately $23 \mathrm{kHz}$ to $14 \mathrm{kHz}$. Again, when reducing the mass flow the levels rise at low frequencies and decrease at high frequencies, in this case the turning point being around $8 \mathrm{kHz}$. BPF peaks are still clearly visible except for the lowest frequency harmonic.

In contrast with the noticeable differences found between the spectra at different air mass flow conditions that were recorded by the inducer probe, the inlet sensor shows an evolution similar to that of the outlet. A clear increase in levels can be seen for frequencies below $5 \mathrm{kHz}$ as the mass flow is reduced, whereas the levels at high frequencies remain similar or even diminish slightly with respect to the high mass flow cases.

The broadband in this case appears to be centred around $7 \mathrm{kHz}$ but, most interestingly, in all the spectra this TCN-like broadband collapses with a deep decrease in level at $5 \mathrm{kHz}$. By using the expression proposed by Eriksson [35] for the onset of the first and second symmetric acoustic modes in ducts with flow:

$$
f_{1}=1.84 \frac{a}{\pi D} \sqrt{1-M^{2}} \quad f_{2}=3.05 \frac{a}{\pi D} \sqrt{1-M^{2}}
$$

these limits were estimated for the inlet and outlet ducts, and have been marked with dotted lines in Fig. 10. As can be seen, the onset of the first mode, and thus the limit of the plane wave range, is around $5 \mathrm{kHz}$ with small differences due the change in temperature, diameter and Mach number from the inlet to the outlet.

While in the case of the inlet duct $f_{1}$ appears to coincide with the abrupt lower bound of the TCN-like broadband and the start of the gradual increase in the levels, the broadband at the outlet has shifted enough so that its lower frequency limit is higher than $f_{1}$ and thus the broadband content is symmetric, without the collapse at the plane wave limit observed at the inlet.

No collapse or influence at the second mode onset frequency can be attested in the outlet case. It becomes thus apparent that while the TCN-like higher frequency broadband propagates above plane wave conditions, it does not appear to be related to the onset of a certain acoustic mode. Apparently, this TCN-like broadband does not propagate below $f_{1}$.

Furthermore, large shifts in the frequency of this broadband noise are demonstrated between the different measurement positions, probably due to the changes in the speed of sound as temperature varies. This makes it difficult to ascribe this phenomenon to a particular flow-related mechanism, as its original frequency is not so clear.

As for the pressure probes, both turning points appear to be more related to the onset of the first mode at $5 \mathrm{kHz}$, but specially in the case of the diffuser it should be taken into account that the given expression for a circular pipe does not apply, and the acoustic modes will depend on the particular geometry of the compressor. 


\subsubsection{Noise}

A simple metric of sound level, used by most authors when measuring turbocharger noise, is the Sound Pressure Level (SPL) $[20,22,36]$. It is a scalar magnitude, and can be calculated from the aforementioned pressure signals as:

$$
\mathrm{SPL}_{\mathrm{dB}}=20 \log _{10}\left(\frac{\bar{P}}{p_{0}}\right)
$$

Here, $\bar{P}$ is the RMS average of the pressure wave amplitude, restricted to the desired frequency range, and $p_{0}$ is a reference pressure value of $2 \cdot 10^{-5} \mathrm{~Pa}$ related to the threshold of human perception.

While useful for free field sound measurements, local SPL measurements have disadvantages in the case of ducts with flow, since as a scalar magnitude it is highly dependent on the transducer position and thus vulnerable to reflections, standing waves and other perturbations associated with the precise duct geometry: consider for instance the differences in Fig. 10 between inducer and inlet duct measurements.

In those cases, it is preferable to compute the Sound Intensity Level as done by instance by Gaudé et al [37]. This is a vector magnitude that characterizes the total sound power travelling along the pipe, which should remain constant save for a small attenuation. Sound intensity in ducts with flow can be calculated by means of the equation proposed by Morfey [38] and applied in $[39,40]$ :

$$
I=I^{+}-I^{-}=\frac{1}{\rho a}\left(\left|\mathbf{X}^{+}\right|^{2}(1+M)^{2}-\left|\mathbf{X}^{-}\right|^{2}(1-M)^{2}\right)
$$

where $\mathbf{X}^{+}$and $\mathbf{X}^{-}$are the spectra of forward and backward pressure waves, $\rho$ and $a$ are the ambient density and sound speed, respectively, and $M$ is the Mach number of the flow.

Several methods exist for performing the decomposition of the pressure signal into its forwards and backwards components, such as the two-microphone method proposed by Seybert [41]. In this work, however, a more sophisticated Linearly Constrained Minimum Covariance beamforming method [42] was selected, which takes advantage of the statistical properties of the signals in order to optimize the signal-to-noise ratio [43, 24].

It should be noted however that while these decomposition techniques are most robust than simple SPL readings, for a completely rig-independant result full two-port characterization should be performed in a rig equipped with active exciters [44]. Also, these methods assume plane wave propagation, so if higher frequencies are required special weighting factors should be used to estimate sound intensity [45].

Once that the intensity is calculated, it is trivial to compute Sound Intensity Level (SIL), using a reference $I_{0}$ of $10^{-12} \mathrm{~W} / \mathrm{m}^{2}$, using:

$$
\mathrm{SIL}_{\mathrm{dB}}=10 \log _{10}\left(\frac{I}{I_{0}}\right)
$$

Following the interpolation procedure described in [23] it is possible to construct "noise charts" that allow for an easy visualization of the variation of the sound intensity level in a given frequency band with the operating condition of the compressor. These charts were calculated for the $1-3 \mathrm{kHz}$ band, and the result is shown in Fig. 11.
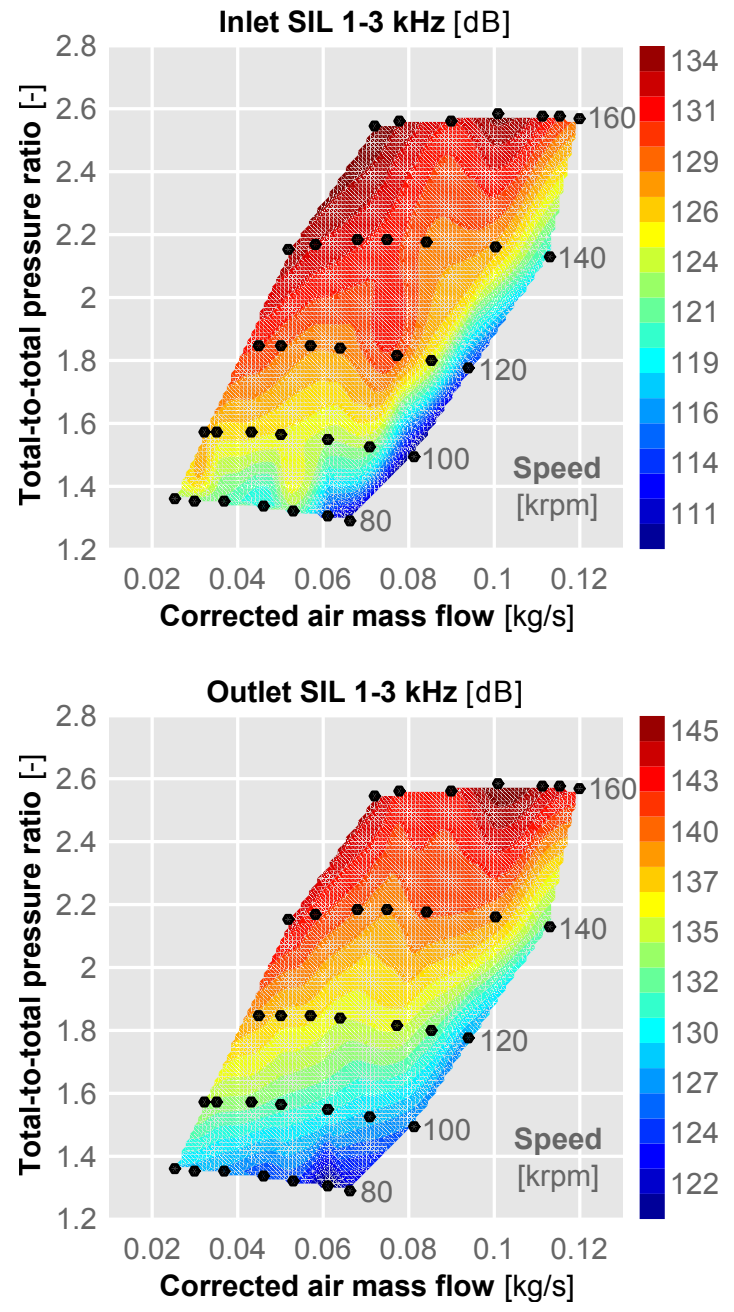

Figure 11: Sound intensity level maps for both inlet (top) and outlet (bottom) ducts in the $1-3 \mathrm{kHz}$ frequency band, interpolated from the measured operating points.

This band was chosen since it is associated with "whoosh" noise [21, 22] typically found in turbocharger compressors. Indeed, as stated previously, a broadband peaking around $2 \mathrm{kHz}$ was found, most notably in the spectra measured by the inducer and diffuser probes at high rotational speeds and low mass flow conditions.

It can be seen in Fig. 11 that sound intensity is higher in the outlet duct than in the inlet duct. Also, at high mass flows where the slope of the iso-speed curve is still changing, low levels are observed for almost all the rotational speeds. However, whereas in both cases the noise levels rise when increasing the rotational speed and decreasing the air mass flow, the distribution is not the same.

Operating conditions with higher level are more extended in the inlet duct, both in terms of rotational speed and mass flow. Except for the maximum rotational speed, rise in noise level occurs in the inlet at a lower flow rate than in the outlet. Consid- 


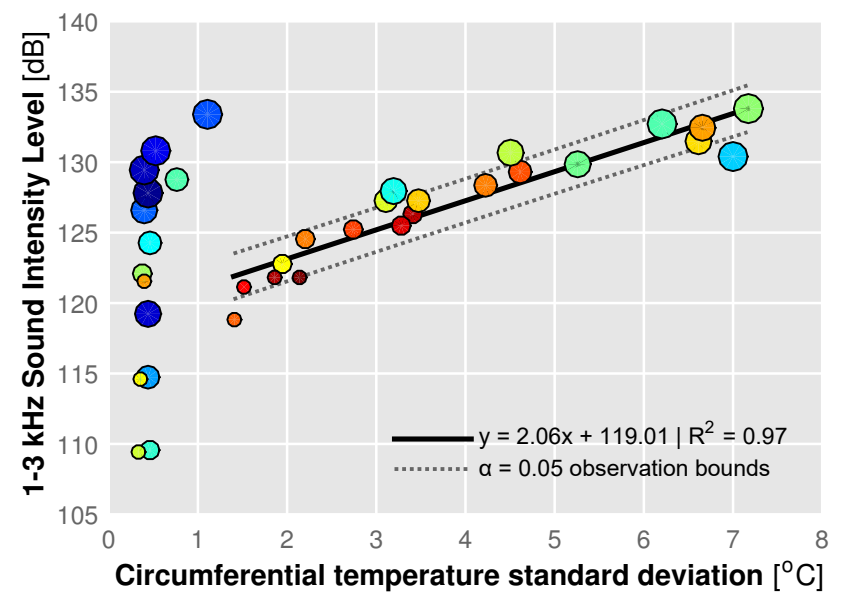

Figure 12: Correlation between standard deviation $\sigma$ at the circumferential thermocouple array and inlet sound intensity level in the $0.7-3 \mathrm{kHz}$ band. Size indicates shaft speed from low to high and color indicates mass flow from higher (blues) to lower (reds).

ering for instance the $140 \mathrm{krpm}$ line, it can be seen that in the inlet, high levels (red colors) are reached just at approximately $80 \mathrm{~g} / \mathrm{s}$ whereas in the outlet red contours are not reached until nearly the deep surge limit at around $60 \mathrm{~g} / \mathrm{s}$.

The slope of the sound intensity contours is also different between inlet and outlet. The level increase is more progressive in the outlet, whereas in the inlet the increase occurs suddenly at mass flows just before the point where the backflow reaches the inlet thermocouples. This suggests that the whoosh broadband noise naturally propagates downstream, but the upstream propagation is promoted only when the backflow extends past the impeller and into the inlet duct. This points to the source flow mechanism being located downstream of the impeller rather than being caused by the instabilities associated with the hot backflow at the inlet duct.

\subsection{Correlations}

In order to further corroborate the existence of a relation between the inlet flow instabilities at marginal surge conditions and the whoosh broadband noise at super-synchronous frequencies, it is possible to correlate the thermal phenomena described above with the total sound level in the relevant frequency range.

Figure 12 shows a plot of inlet sound intensity level against the standard deviation of the temperature in the circumferential thermocouple array. It can be seen that for high temperature deviations the sound intensity appears to be correlated following an ascending trend. However, below approximately $1.1^{\circ} \mathrm{C}$ of deviation the sound level appears to still increase with rotational speed at low mass flows, even if no hot backflow is being detected by the circumferential thermocouple array.

A least squares linear fitting was performed, showing that there is a good correlation between the increase in sound intensity at the selected frequency band and the increase in the skewness of the inlet circumferential temperature distribution, thereby suggesting a link between the two phenomena. Together with the fact that the increase in sound level occurs even at operating conditions at which the backflow producing the temperature

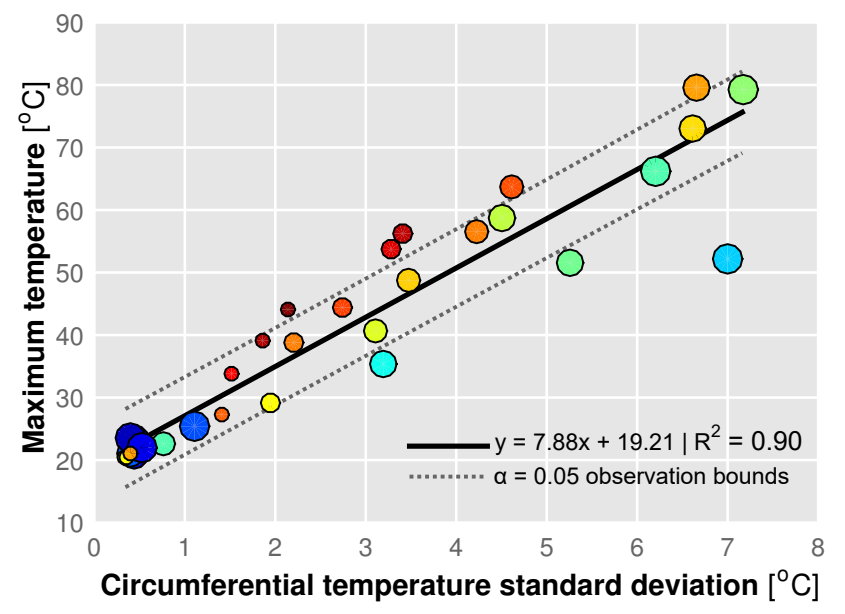

Figure 13: Correlation between standard deviation $\sigma$ at the circumferential thermocouple array and maximum temperature measured by the thermocouples. Size indicates shaft speed from low to high and color indicates mass flow from higher (blues) to lower (reds).

distribution skewness has not yet reached the thermocouples, this again points to the generation of whoosh occurring in or downstream of the impeller, with the phenomena being amplified upstream of the impeller as the backflow extends into the inlet.

Another linear correlation was performed between the circumferential standard deviation of temperature and the maximum temperature of the backflow, as shown in Fig. 13. The good agreement of this correlation showed that a relative or dimensionless standard deviation (this is, raw standard deviation divided by a representative temperature) is approximately constant: the backflow is skewed similarly along the compressor chart and increases linearly with the increase of temperature caused by higher pressure ratios.

This skewness in the circumferential distribution of temperature could be related to the loss of axisymmetry caused by the presence of the volute tongue of the compressor, and its influence on the flow behaviour in the diffuser could constitute a subject worthy of further investigation.

\section{Conclusions}

In this paper, an experimental study is described with the objective of accurately characterizing the behaviour of the inlet flow instability of a turbocharger compressor when marginal surge conditions are reached. Specifically, focus has been put on the high-temperature compressed flow that reverses direction and extends upstream of the impeller and into the final section of the inlet duct after the partial blade stalling that causes these marginal surge conditions begins.

The rotation, vortex shedding and reintegration of this backflow into the main flow coming through the core of the inlet duct have been regarded as possible sources of acoustic noise and several methods such as ported shrouds and different casing treatments have been proposed to alleviate its occurrence and extension $[46,5]$. 
During this investigation the setup described in section 2 has been used to measure the distribution of temperature associated with marginal surge, i.e. conditions with low air mass flow rate, though not low enough to induce the compressor to enter into deep surge.

By using the two thermocouple arrays installed in the compressor inlet, both axial and circumferential temperature measurements were obtained for several operating conditions. A test was also performed in order to characterize the radial distribution of temperature caused by the hot backflow. The robustness of these internal flow measurements against radiation from the inlet pipe walls was verified through external thermographic imaging.

The analysis of these temperature distributions allowed the identification of the backflow length for the whole compressor chart, together with the maximum temperature rise in the inlet and also the skewness of the circumferential temperature distribution of the flow.

The air mass flow has been shown to be the most influencing factor when predicting how far the hot backflow extends upstream of the compressor impeller. At high rotational speeds, where surge occurs at relatively high mass flows, the backflow temperature is higher (due to higher compression ratio) but the length of the backflow is shorter. As for circumferential skewness, it has been shown to increase linearly with backflow temperature, while it appears to be similar in shape for all the different operating conditions considered.

In order to gain further understanding of the generation and evolution of the local flow behaviour, miniaturized pressure probes were placed in the compressor inducer and diffuser. Analysis of the data provided by these two probes, and specially by the former, showed that the pressure spectral information in the inducer abruptly changed when the backflow reached the upstream side of the impeller and the probe, as predicted with the aforementioned temperature data.

Furthermore, compressor inlet and outlet ducts were fitted with arrays of three piezoelectric pressure sensors in order to investigate the well-known mid-frequency acoustical phenomenon usually referred to as whoosh noise. This phenomenon was detected at plane wave frequencies by both probes, even at stable conditions of higher flow rate where hot backflow was not yet detected by the thermocouples This suggests that the unstable structures of recirculating backflow in the inducer and inlet are not the primary source mechanism of whoosh.

As for the other phenomenon usually identified in turbocharger noise spectra, the so-called TCN broadband at higher frequency, it was demonstrated through the experimental results that this broadband content is highly dependent on the particular conditions at each measurement location, and that it appears even at frequencies above the BPF.

It was however noted that when this broadband shifted enough to reach the onset of the first acoustic mode it collapsed precisely on this limit and did not propagate at plane wave frequencies, unlike whoosh noise that appears precisely in this range. This provides a clear way to differentiate between these two broadband phenomena, a distinction that sometimes is absent in some works of the literature.
A correlation between sound intensity in the whoosh characteristic frequency band and the increase in temperature skewness due to the backflow was also found. Comparison of inlet and outlet sound intensity charts shows a more smooth evolution in the outlet and a more sudden rise in the inlet, linked to conditions where the backflow starts to appear.

In conjunction with the analysis of inlet temperature and pressure data, this suggests that whoosh noise in the inlet is boosted by the convective effect of the reversed flow structures at the inlet in marginal surge conditions, but it is not caused by them.

Still, strategies aiming at the control of these backflow structures could influence noise transmission on the downstream side and potentially alleviate its adverse effects on vehicle NVH perception. These strategies could also address coupling effects between the fluid structures and the structural modes of the duct that increase radiated noise.

Further research combining the experimental gathering of pressure data inside the compressor volute and/or the use of numerical CFD models is, however, needed to precisely pinpoint and mitigate the source of this adverse phenomenon, and not only its propagation.

\section{Acknowledgements}

This work has been partially supported by Jaguar Land Rover Limited, Abbey Road, Whitley, Coventry CV3 4LF, UK.

The equipment used in this work has been partially supported by the Spanish Ministerio de Economía y Competitividad through the grant $n^{\circ}$ DPI2015-70464-R and by FEDER project funds "Dotación de infraestructuras científico técnicas para el Centro Integral de Mejora Energética y Medioambiental de Sistemas de Transporte (CiMeT), (FEDER-ICTS-2012-06)" framed in the operational program of unique scientific and technical infrastructure of the Spanish Ministerio de Economía y Competitividad.

J. García-Tíscar is partially supported through contract FPIS2-2015-1530 of the Programa de Apoyo para la Investigación y Desarrollo (PAID) of Universitat Politècnica de València.

The authors also wish to thank Mr. Raj Kumar Sharma for his valuable contributions and Mr. Bernardo Planells for his inestimable assistance during the experimental campaign.

\section{References}

[1] F. Schumann, F. Sarikoc, S. Buri, H. Kubach, U. Spicher, Potential of spray-guided gasoline direct injection for reduction of fuel consumption and simultaneous compliance with stricter emissions regulations, International Journal of Engine Research 14 (1) (2013) 80-91. doi: 10.1177/1468087412451695.

[2] C. Teng, S. Homco, Investigation of Compressor Whoosh Noise in Automotive Turbochargers, SAE Int. J. of Passeng. Cars-Mech. Syst. 2 (1) (2009) 1345-1351. doi:10.4271/2009-01-2053.

[3] I. J. Day, Stall, surge, and 75 years of research, Journal of Turbomachinery 138 (1) (2016) 011001. doi:10.1115/1.4031473.

[4] R. O. Bullock, W. W. Wilcox, J. J. Moses, Experimental and theoretical studies of surging in continuous-flow compressors, Tech. rep., NACA (1946).

URL NationalAdvisoryCommitteeforAeronautics 
[5] L. Ding, T. Wang, B. Yang, W. Xu, C. Gu, Experimental investigation of the casing treatment effects on steady and transient characteristics in an industrial centrifugal compressor, Experimental Thermal and Fluid Science 45 (2013) 136-145. doi:10.1016/j . expthermflusci. 2012 10.016.

[6] J. Galindo, J. R. Serrano, X. Margot, A. Tiseira, N. Schorn, H. Kindl, Potential of flow pre-whirl at the compressor inlet of automotive engine turbochargers to enlarge surge margin and overcome packaging limitations, International journal of heat and fluid flow 28 (3) (2007) 374-387. doi: 10.1016/j.ijheatfluidflow.2006.06.002.

[7] J. Galindo, H. Climent, C. Guardiola, A. Tiseira, On the effect of pulsating flow on surge margin of small centrifugal compressors for automotive engines, Experimental Thermal and Fluid Science 33 (8) (2009) 1163 1171. doi:10.1016/j.expthermflusci.2009.07.006.

[8] W. R. Hawthorne, Some aerodynamic problems of aircraft engines, Journal of the Aeronautical Sciences 24 (10) (1957) 713-730. doi:10.2514/8 3954.

[9] L. Wang, C. Yang, B. Zhao, D. Lao, C. Ma, D. Li, The change of the inlet geometry of a centrifugal compressor stage and its influence on the compressor performance, Journal of Thermal Science 22 (3) (2013) 197 208. doi:10.1007/s11630-013-0613-2. URL http://dx.doi.org/10.1007/s11630-013-0613-2

[10] H. Stoffels, M. Schroeer, NVH Aspects of a Downsized Turbocharged Gasoline Powertrain with Direct Injection, SAE Technical Paper 2003-011664. doi : 10.4271/2003-01-1664.

[11] A. Miśkiewicz, T. Letowski, Psychoacoustics in the automotive industry, Acta Acustica united with Acustica 85 (5) (1999) 646-649.

URL http://www.ingentaconnect.com/content/dav/aaua/ 1999/00000085/00000005/art00019

[12] J. M. Desantes, A. J. Torregrosa, A. Broatch, Experiments on flow noise generation in simple exhaust geometries, Acta Acustica united with Acustica 87 (1) (2001) 46-55.

URL http://www.ingentaconnect.com/content/dav/aaua/ 2001/00000087/00000001/art00007

[13] J. Galindo, J. R. Serrano, C. Guardiola, C. Cervelló, Surge limit definition in a specific test bench for the characterization of automotive turbochargers, Experimental Thermal and Fluid Science 30 (5) (2006) 449-462. doi: 10.1016/j.expthermflusci.2005.06.002.

[14] S. Koff, E. Greitzer, Axisymmetrically stalled flow performance for multistage axial compressors, Journal of Turbomachinery 108 (2) (1986) 216-223. doi:10.1115/1.3262040.

[15] J. Galindo, J. Serrano, H. Climent, A. Tiseira, Experiments and modelling of surge in small centrifugal compressor for automotive engines, Experimental Thermal and Fluid Science 32 (3) (2008) 818-826. doi: 10.1016/j.expthermflusci.2007.10.001.

[16] A. Liu, X. Zheng, Methods of surge point judgment for compressor experiments, Experimental Thermal and Fluid Science 51 (2013) 204-213. doi:10.1016/j.expthermflusci.2013.07.015.

[17] R. Lang, Contribución a la Mejora del Margen de Bombeo en Compresores Centrífugos de Sobrealimentación, Ph.D. thesis, Universitat Politècnica de València (2011). doi: 10.4995/Thesis/10251/12331.

[18] J. Andersen, F. Lindström, F. Westin, Surge definitions for radial compressors in automotive turbochargers, SAE International Journal of Engines 1 (1) (2009) 218-231. doi : 10.4271/2008-01-0296.

[19] N. Figurella, R. Dehner, A. Selamet, K. Tallio, K. Miazgowicz, R. Wade, Noise at the mid to high flow range of a turbocharger compressor, Noise Control Engineering Journal 62 (5) (2014) 306-312. doi:10.3397/1/ 376229.

[20] D. Evans, A. Ward, The reduction of turbocharger whoosh noise, in: Proceedings of the 8 th International Conference on Turbochargers and Turbocharging, London, UK, 2006, pp. 29-42. doi:10.1016/ B978-1-84569-174-5.50006-3.

[21] D. Evans, A. Ward, Minimizing Turbocharger Whoosh Noise for Diesel Powertrains, SAE Technical Paper 2005-01-2485. doi:10.4271/ 2005-01-2485.

[22] E. P. Trochon, A new type of silencers for turbocharger noise control, SAE Technical Paper 110 (6) (2001) 1587-1592. doi:10.4271/ 2001-01-1436.
[23] A. J. Torregrosa, A. Broatch, X. Margot, J. García-Tíscar, Experimental methodology for turbocompressor in-duct noise evaluation based on beamforming wave decomposition, Journal of Sound and Vibration 376 (2016) 60-71. doi:10.1016/j.jsv.2016.04.035.

[24] G. Piñero, L. Vergara, J. M. Desantes, A. Broatch, Estimation of velocity fluctuation in internal combustion engine exhaust systems through beamforming techniques, Measurement Science \& Technology 11 (11) (2000) 1585-1595. doi:10.1088/0957-0233/11/11/307.

[25] A. Broatch, J. Galindo, R. Navarro, J. García-Tíscar, Numerical and experimental analysis of automotive turbocharger compressor aeroacoustics at different operating conditions, International Journal of Heat and Fluid Flowdoi:10.1016/j.ijheatfluidflow.2016.04.003.

[26] A. Broatch, J. Galindo, R. Navarro, J. García-Tíscar, A. Daglish, R. Sharma, Simulations and measurements of automotive turbocharger compressor whoosh noise, Engineering Applications of Computational Fluid Mechanics 9 (1) (2015) 12-20. doi:10.1080/19942060.2015. 1004788.

[27] A. Broatch, J. Galindo, R. Navarro, J. García-Tíscar, Methodology for experimental validation of a CFD model for predicting noise generation in centrifugal compressors, International Journal of Heat and Fluid Flow 50 (2014) 134 - 144. doi:10.1016/j.i jheatfluidflow.2014.06.006.

[28] J. Galindo, A. Tiseira, F. J. Arnau, R. Lang, On-engine measurement of turbocharger surge limit, Experimental Techniques 37 (1) (2013) 47-54. doi:10.1111/j.1747-1567.2010.00697.x.

[29] D. Fink, N. Cumpsty, E. Greitzer, Surge dynamics in a free-spool centrifugal compressor system, Journal of Turbomachinery 114 (2) (1992) 321-332. doi:10.1115/1.2929146.

[30] T. Raitor, W. Neise, Sound generation in centrifugal compressors, Journal of Sound and Vibration 314 (2008) 738 - 756. doi:10.1016/j.jsv . 2008.01.034.

[31] F. Kameier, W. Neise, Rotating blade flow instability as a source of noise in axial turbomachines, Journal of Sound and Vibration 203 (5) (1997) 833-853. doi:10.1006/jsvi.1997.0902.

[32] H. Tiikoja, H. Rämmal, M. Abom, H. Boden, Investigations of automotive turbocharger acoustics, SAE International Journal of Engines 4 (2) (2011) 2531-2542. doi:10.4271/2011-24-0221.

[33] J. Galindo, A. Tiseira, R. Navarro, M. López, Influence of tip clearance on flow behavior and noise generation of centrifugal compressors in nearsurge conditions, International Journal of Heat and Fluid Flow 52 (2015) 129-139. doi:10.1016/j.ijheatfluidflow.2014.12.004.

[34] G. Liśkiewicz, L. Horodko, M. Stickland, W. Kryłłowicz, Identification of phenomena preceding blower surge by means of pressure spectral maps, Experimental Thermal and Fluid Science 54 (2014) 267-278. doi : 10.1016/j . expthermflusci.2014.01.002.

[35] L. J. Eriksson, Higher order mode effects in circular ducts and expansion chambers, Journal of the Acoustical Society of America 68 (1980) 545 doi: $10.1121 / 1.384768$.

[36] Y. Lee, D. Lee, Y. So, D. Chung, Control of Airflow Noise From Diesel Engine Turbocharger, SAE Technical Paper (2011-01-0933). doi:10. 4271/2011-01-0933.

[37] G. Gaudé, T. Lefèvre, R. Tanna, K. Jin, T. J. B. McKitterick, S. Armenio, Experimental and computational challenges in the quantification of turbocharger vibro-acoustic sources, in: Proceedings of the 37th International Congress and Exposition on Noise Control Engineering (INTER-NOISE 2008), Vol. 2008, Institute of Noise Control Engineering, 2008, pp. 5754 5767.

URL http://www.ingentaconnect.com/content/ince/incecp/ 2008/00002008/00000003/art00009

[38] C. L. Morfey, Sound transmission and generation in ducts with flow, Journal of Sound and Vibration 14 (1) (1971) 37-55. doi:10.1016/ 0022-460X (71) 90506-2.

[39] E. Dokumaci, On calculation of acoustic power, Journal of Sound and Vibration 238 (5) (2000) 869-876. doi:10.1006/jsvi.2000.3138.

[40] K. Holland, P. Davies, The measurement of sound power flux in flow ducts, Journal of Sound and Vibration 230 (4) (2000) 915-932. doi : 10.1006/jsvi.1999. 2656.

[41] A. F. Seybert, Two-sensor methods for the measurement of sound intensity and acoustic properties in ducts, Journal of the Acoustical Society of America 83 (6) (1988) 2233-2239. doi:10.1121/1.396352. 
[42] O. Frost, An algorithm for linearly constrained adaptive array processing, Proceedings of the IEEE 60 (8) (1972) 926-935. doi : 10.1109/PROC 1972.8817.

[43] B. Van Veen, K. Buckley, Beamforming: A versatile approach to spatial filtering, ASSP Magazine, IEEE 5 (2) (1988) 4-24. doi:10.1109/53. 665.

[44] R. Kabral, H. Rammal, M. Abom, Acoustical methods for investigating turbocharger flow instabilities, SAE Technical Paper 2013-01-1879. doi: 10.4271/2013-01-1879.

[45] A. Hynninen, M. Åbom, Determination of in-duct sound power beyond the plane wave range using wall-mounted microphones, Applied Acoustics 99 (2015) 24 - 30. doi:10.1016/j . apacoust.2015.05.003.

[46] H. Chen, V. Lei, Casing treatment and inlet swirl of centrifugal compressors, in: Proceedings of ASME Turbo Expo 2012, no. GT2012-69340, ASME, 2012. doi:10.1115/1.4007739. 